\title{
Evaluation and improvement the safety of total marrow irradiation with helical tomotherapy using repeat failure mode and effects analysis
}

Jiuling Shen ${ }^{1,2+}$, Xiaoyong Wang ${ }^{1,2+}$, Di Deng ${ }^{1,2}$, Jian Gong ${ }^{1,2}$, Kang Tan ${ }^{1,2}$, Hongli Zhao ${ }^{1,2}$, Zhirong Bao ${ }^{1,2}$, Jinping Xiao ${ }^{1,2}$, An Liu ${ }^{3}$, Yunfeng Zhou ${ }^{1,2}$, Hui Liu ${ }^{1,2^{*}}$ and Conghua Xie ${ }^{1,2^{*}}$

\begin{abstract}
Background \& purpose: Helical tomotherapy has been applied to total marrow irradiation (HT-TMI). Our objective was to apply failure mode and effects analysis (FMEA) two times separated by 1 year to evaluate and improve the safety of HT-TMI.

Materials and methods: A multidisciplinary team was created. FMEA consists of 4 main steps: (1) Creation of a process map; (2) Identification of all potential failure mode (FM) in the process; (3) Evaluation of the occurrence (O), detectability (D) and severity of impact (S) of each FM according to a scoring criteria (1-10), with the subsequent calculation of the risk priority number (RPN=O*D*S) and (4) Identification of the feasible and effective quality control (QC) methods for the highest risks. A second FMEA was performed for the high-risk FMs based on the same risk analysis team in 1 year later.

Results: A total of 39 subprocesses and 122 FMs were derived. First time RPN ranged from 3 to 264.3. Twenty-five FMs were defined as being high-risk, with the top 5 FMs (first RPN/ second RPN): (1) treatment couch movement failure (264.3/102.8); (2) section plan dose junction error in delivery (236.7/110.4); (3) setup check by megavoltage computed tomography (MVCT) failure (216.8/94.6); (4) patient immobilization error (212.5/90.2) and (5) treatment interruption (204.8/134.2). A total of 20 staff members participated in the study. The second RPN value of the top 5 high-risk FMs were all decreased.
\end{abstract}

Conclusion: QC interventions were implemented based on the FMEA results. HT-TMI specific treatment couch tests; the arms immobilization methods and strategy of section plan dose junction in delivery were proved to be effective in the improvement of the safety.

Keywords: Failure mode and effects analysis, Total marrow irradiation, Helical tomotherapy, Process quality assurance

\footnotetext{
*Correspondence: hbzkznyy@163.com; chxie_65@whu.edu.cn

${ }^{\dagger}$ Jiuling Shen and Xiaoyong Wang contributed equally to this work.

'Department of Radiation and Medical Oncology, Zhongnan Hospital of

Wuhan University, 169 Donghu Road, Wuhan, Hubei 430070, People's

Republic of China

Full list of author information is available at the end of the article
}

(C) The Author(s). 2019 Open Access This article is distributed under the terms of the Creative Commons Attribution 4.0 International License (http://creativecommons.org/licenses/by/4.0/), which permits unrestricted use, distribution, and reproduction in any medium, provided you give appropriate credit to the original author(s) and the source, provide a link to the Creative Commons license, and indicate if changes were made. The Creative Commons Public Domain Dedication waiver (http://creativecommons.org/publicdomain/zero/1.0/) applies to the data made available in this article, unless otherwise stated. 


\section{Introduction}

Total marrow irradiation (TMI) has been an important part of conditioning regimens for patients undergoing hematopoietic cell transplantation [1-3]. TMI treated with helical tomotherapy (HT-TMI) have been proved to be a clinically feasible way with a uniform and accurate dose distribution to the target and a significant reduction in side effects [4-8]. The long irradiation target volume makes the process much more complex compared to the conventional radiotherapy for tumors. Any potential errors can lead to serious treatment failure and tighter tolerances should be placed on QC methods. In addition to implement tighter tolerances of the routine daily and monthly equipment-related QC, the importance of human factors should not be ignored [9-11]. Although, many centers have published their application of HT-TMI, Unfortunately, no one has reported the safety and QC of HT-TMI process, and the existing potential errors haven't been figured out and analyzed, which could be very dangerous and imperative.

FMEA as a prospective risk method has been applied to analyze radiotherapy processes by Scorsetti [12] first, and proved to be an effective method for quality improvement [13-21]. FMEA was organised into four main steps: (1) process analysis, (2) identification of the FMs, (3) risk analysis, (4) identification of corrective actions. In our study, the score team consists of a manager of the Medical Quality Control Committee of our department, a chief physicist and a chief therapist and the regular physician, physicist and therapist who have obtained a qualification certificate and participated in the daily clinical treatment process with a range of number of years experience.

\section{Materials and methods}

\section{HT-TMI technique}

The HT-TMI program at our institution started in 2016. More than 80 patients have been treated. The process of HT-TMI is completed in 1 week, with a total prescription of
8 Gy delivered by 2 fractions (4 Gy per fraction) separated by $\geq 6 \mathrm{~h}$ in 1 day. As is shown in Fig. 1a, all patients were immobilized using a home-made dedicated immobilization system. CT scans, treatment planning and delivery details can be found in our previous study [22].

\section{FMEA process}

A multidisciplinary risk analysis team was established consisting of two nurses, five medical physicists, four physician and nine therapists (details shown in Table 1). The team was led by a manager of the Medical Quality Control Committee of our department, and all members were involved in the scoring process. The FMEA was performed in several steps (in order):

1. Every step in the process was identified by each member with regard to his or her own area of expertise. Because of the level of details of each step was found to be different among members, discussion was carried until the team reached a consensus on the steps within each subprocess. The goal was to ensure each step was clear and manageable. After that, all steps were wrote down and laid out in the order to build the process tree.

2. Identification of failure modes (FM) for each step as many as the team could imagine. The possible causes and clinical situations where the failure could occur as well as $\mathrm{S}$ were listed with their collective experience.

3. Each FM was assigned three scores $(\mathrm{O}, \mathrm{D}$ and $\mathrm{S})$ of 1 to 10 with a consensus 10-point scale (shown in Table 2) based on their individual experiences. RPN value was derived by multiplying the three individual scores with each other. The averaged O, D, RPN value and maximum $\mathrm{S}$ score of all members was defined as the final score. FMs were ranked by RPNs from highest to lowest, the top $20 \%$ were defined as "high-risk".

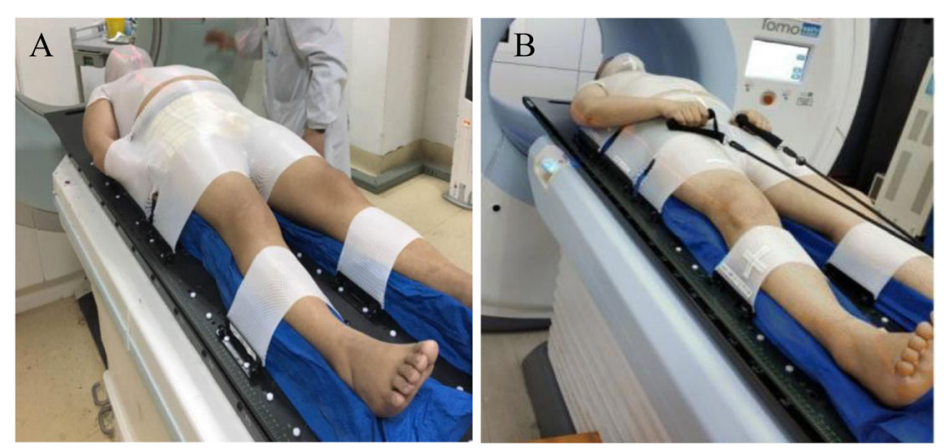

Fig. 1 Example of the use of the 3 thermoplastic masks for patient immobilization. a first immobilization system and b new immobilization system 
Table 1 Details in the composition of the scoring team, the total number of scorers and the number of scorers with different working years in each sub-process

\begin{tabular}{|c|c|c|c|c|c|}
\hline \multirow[t]{2}{*}{ Sub_process } & \multirow[t]{2}{*}{ Members } & \multicolumn{3}{|c|}{$\begin{array}{l}\text { Number of scorers with } \\
\text { different years of experience }\end{array}$} & \multirow[t]{2}{*}{ Total } \\
\hline & & $1-3$ years & $3-5$ years & $>5$ years & \\
\hline Registration & Nurse & 0 & 1 & 1 & 2 \\
\hline $\begin{array}{l}\text { Immobilization /CT simulation } \\
\text { Image transmission }\end{array}$ & Therapist & 1 & 1 & 2 & 4 \\
\hline $\begin{array}{l}\text { Contouring /Prescription } \\
\text { Plan approval }\end{array}$ & Physician & 2 & 1 & 1 & 4 \\
\hline $\begin{array}{l}\text { Treatment planning/evaluation } \\
\text { Plan preparation }\end{array}$ & Physicist & 2 & 2 & 1 & 5 \\
\hline Treatment preparation/delivery & Therapist & 2 & 2 & 1 & 5 \\
\hline
\end{tabular}

4. Fault tree analysis was performed to visualize the locations and propagation of FMs through the process. Appropriate quality management (QM) program were implemented to mitigate the most important risks which were identified in the previous analyses.

5. Second FMEA was performed for the high-risk FMs follow the steps 3 above based on the same risk analysis team in 1 year later to verify the effectiveness of the QM program.

\section{Results}

HT-TMI process map is shown in Fig. 2. Thirty-nine subprocess and 122 FMs were identified with a total of 20 members participating.

In the first time FMEA, RPN values range from 3 to 264.3 with a median value 30 and average value 42 , the average value of $\mathrm{O}, \mathrm{D}$ and $\mathrm{S}$ were $2.0,2.3$ and 4.8, respectively. A total of 25 high-risk FMs were found, and top 5 FMs (first RPN/ second RPN) are (details shown in Table 3): (1) treatment couch movement failure (264.3/102.8); (2) section plan dose junction error in delivery (236.7/110.4); (3) setup check by MVCT failure (216.8/94.6); (4) patient immobilization error (212.5/
90.2) and (5) treatment interruption (204.8/134.2). The RPN value of the top 5 high-risk FMs found in the first FMEA were all decreased and no longer high-risk FMs in the second FMEA after adaption of the QM program. A total of 20 new high-risk FMs were found and appropriate QM program were implemented.

\section{Treatment couch movement failure}

The delivery of a HT-TMI plan requires the accuracy of table movement with approximately $160 \mathrm{~cm}$ in length. Since the helical tomotherapy system was commissioned, the couch tests were performed on a monthly basis followed by the recommendation of AAPM TG148 [23]. During the delivery of HT-TMI, the subtle couch movement error was very difficult to detect by the traditional test with only a movement distance of $20 \mathrm{~cm}$, so the team gave a D score 5.2 and the highest RPN value 264.3. After group discussion, the test of the couch was redesigned, the measuring distance is extended from the previous 20 to $160 \mathrm{~cm}$, the tolerance between physical distances traveled and the digital readout at different couch heights was set to be within $3 \mathrm{~mm}$, the leveling of the stationary couch should be less than $0.5^{\circ}$ and the lateral couch position should deviate by less than $3 \mathrm{~mm}$. In

Table 2 Discription of 10-point scales used to derive values of probability, severity, and detectability for each failure mode

\begin{tabular}{|c|c|c|c|}
\hline Rank & Occurence(O) & Severity(S) & Detectability(D) \\
\hline 1 & \multirow{2}{*}{$\begin{array}{l}\text { Very low; } \\
\text { less than } 2 \%\end{array}$} & No effect & \multirow[t]{2}{*}{ Very easy to detect; alomost never miss } \\
\hline 2 & & Inconvenience; & \\
\hline 3 & \multirow{2}{*}{$\begin{array}{l}\text { Low; } \\
\text { approximately } \\
5 \%\end{array}$} & discomfort or distress & \multirow[t]{2}{*}{ Easy to detect, double check should be performed } \\
\hline 4 & & $\begin{array}{l}\text { Minor dosimetric error; } \\
\text { approximately } 5 \%\end{array}$ & \\
\hline 5 & Moderate & \multirow{2}{*}{$\begin{array}{l}\text { Limited toxicity or underdose; } 10 \% \text { dose } \\
\text { difference with insufficient accutacy }\end{array}$} & Moderate, a "lucky catch" \\
\hline $\begin{array}{l}6 \\
7\end{array}$ & $\begin{array}{l}\text { Medium; } \\
\text { occasional failure }\end{array}$ & & \multirow[t]{2}{*}{$\begin{array}{l}\text { Very difficult to detect a subtle error in mechanical position or highly } \\
\text { unusual situation, e.g., treatment table auto setup error }\end{array}$} \\
\hline 8 & \multirow{2}{*}{$\begin{array}{l}\text { High; } \\
\text { repeated failures }\end{array}$} & & \\
\hline 9 & & Very serious toxicity; patient injury or death & Almost impossible to detect \\
\hline 10 & Inevitable & & \\
\hline
\end{tabular}




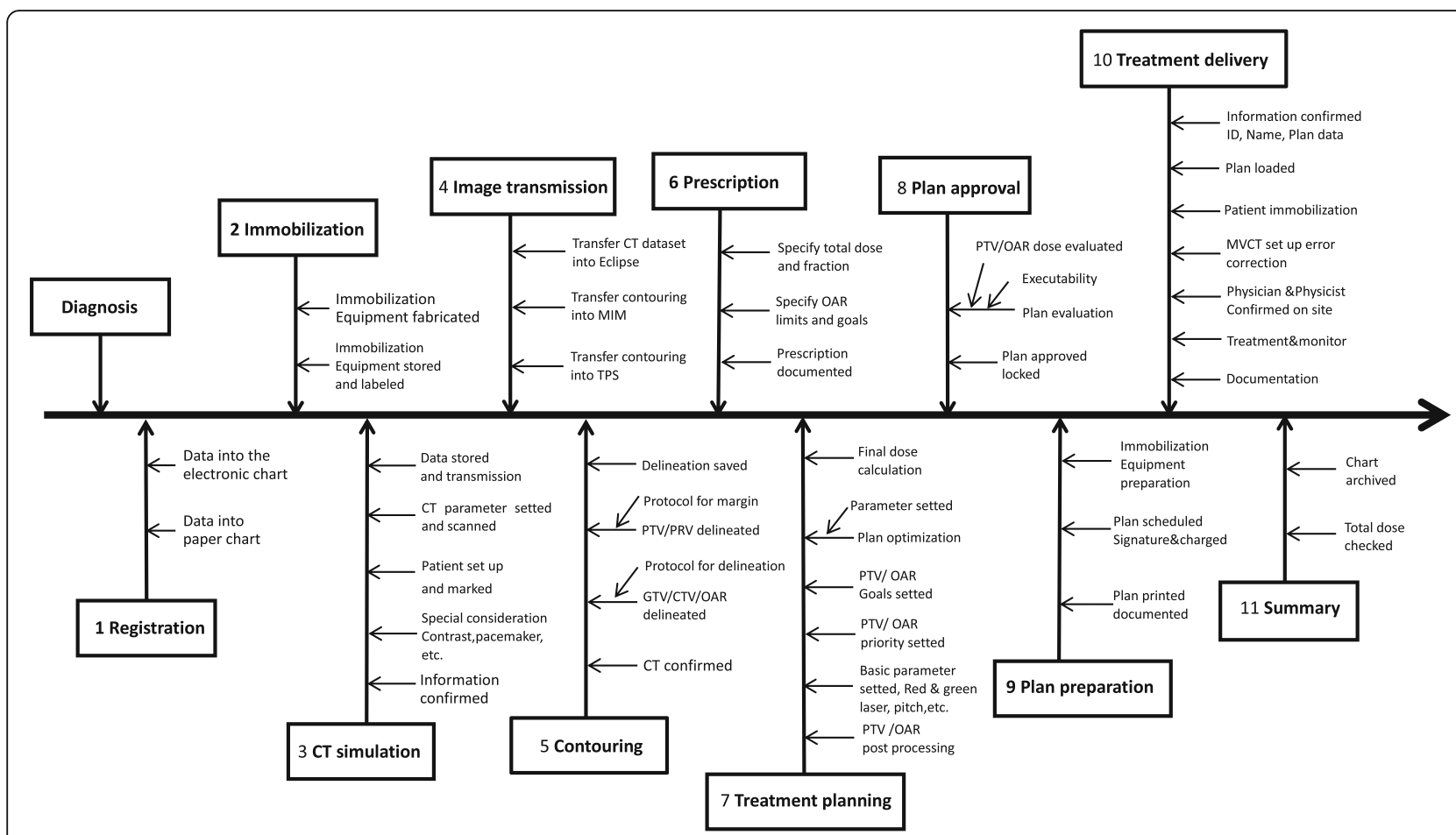

Fig. 2 Process map of total marrow irradiation with helical tomotherapy

addition, the test frequency from the previous monthly becomes the measurement before each treatment.

\section{Section plan dose junction error in delivery}

Dose homogeneity at the junction region from the overlap of the two plans was considered in the treatment planning stage [22]. In the delivery stage, we have performed three MVCT scans in Plan-upper and one for Plan-lower [22], but no scans covered the dose junction region, after couch shifts of the fourth MVCT scans, the three consecutive slices (used for section plan dose junction) from the Plan-upper and Plan-lower may mismatch. Resulting in hot-spots and cold-spots near the junction region. After group discussion, we demand that an additional MVCT scan covering the dose junction region must be performed in the delivery of Plan-upper, and the longitudinal distance of the fiducial markers between planning CT and MVCT is measured. To complement the dose distributions of junction region from
Plan-upper, in the Plan-lower delivery stage, the shifts in SI direction were set to the opposite value (Plan-lower is delivered in FFS) of the measured distance above to match the setup error in SI direction from Plan-upper.

\section{Setup check by MVCT failure}

In HT-TMI, three MVCT scans and segmented treatments for the Plan-upper were implemented to guarantee the treatment accuracy. However, considering factors such as the dose uniformity of the interruption position, the MVCT scan dose and efficiency of delivery, it was sometimes necessary to follow the principle of reducing the segmented treatments as much as possible when the patient was well immobilized and setup was precise. Sometimes the whole body setup check (assessment of the whole body setup deviation) by MVCT in the early setup stage was not performed by therapists who expected that the later three MVCT scans could eliminate all the setup errors in LR, SI and AP directions, as well

Table 3 Top 5 failure mode, occurrence (O), detectability (D) and risk priority number (RPN), subscripts 1 and 2 indicate the first time and second time scores respectively

\begin{tabular}{|c|c|c|c|c|c|c|}
\hline NO. & Sub_process & FM & $\mathrm{O}_{1} / \mathrm{O}_{2}$ & $D_{1} / D_{2}$ & $S_{1} / S_{2}$ & $\mathrm{RPN}_{1} / \mathrm{RPN}_{2}$ \\
\hline 1 & Treatment delivery & Treatment couch movement failure & $5.2 / 4.8$ & $6.5 / 3.2$ & $8 / 8$ & $264.3 / 102.8$ \\
\hline 2 & Treatment delivery & Section plan dose junction error in delivery & $4.8 / 4.5$ & $5.6 / 3.1$ & $9 / 9$ & $236.7 / 110.4$ \\
\hline 3 & Treatment delivery & Setup check by MVCT failure & $6.5 / 3.3$ & $4.5 / 3.5$ & $7 / 7$ & 216.8/94.6 \\
\hline 4 & Immobilization & Patient immobilization error & $7.5 / 3.2$ & $3.6 / 3.6$ & $8 / 8$ & $212.5 / 90.2$ \\
\hline 5 & Treatment delivery & Treatment interruption & $8.5 / 5.5$ & $3.2 / 3.2$ & $7 / 7$ & 204.8/134.2 \\
\hline
\end{tabular}


as rotation around the SI axis. But in fact, if the patient was not setup perfectly, especially rotation around the AP axis, often more than three scans thus two interruptions (i.e., over-scan and over-interruption) must be performed to reduce the setup errors in the treatment stage. That resulted in an increased scanning dose, a risk of underdose or overdose near the interruption position and a prolonged treatment time. So the risk team gave the setup check by MVCT failure a RPN value of 216.8 . With these concerns, we demand that therapists must perform three short scans (scan 1, orbits to the first cervical vertebra $(\mathrm{C} 1)$; scan 2 , seventh thoracic vertebra (T7 to T10); scan 3, fifth lumbar vertebra (L5) to sacrum.) to check the whole body setup deviation by MVCT after setup. If the difference between the three scans is more than $5 \mathrm{~mm}$ in any of the three translation directions and $1^{\circ} \mathrm{in}$ roll, the patient needs to be re-setup again.

\section{Patient immobilization error}

Before first FMEA, shoulders and arms of the patients were immobilized close to both sides of the body and all covered in the masks. During treatment, if the whole body's transverse diameter exceeded $40 \mathrm{~cm}$, part of the shoulders and arms could not be fully captured by MVCT, because of the max field-of-view of HT-MVCT is only $40 \mathrm{~cm}$ in diameter. Therefore, the PTVs in the shoulders and arms were outside of the field-view where could not be checked precisely. After first FMEA, we developed a new method of immobilizing the shoulders and arms. Briefly, shoulders of the patients were immobilized close to both sides of the body and covered by the first mask, arms were positioned close to the thorax outside of the second mask; while hands were positioned on the groins with fingers grasping the rope to ensure good reproducibility (Fig. 1b). In this way, most of the PTVs in shoulders and arms could be seen in the MVCT images with only a little lateral soft tissue in the shoulders outside of the MVCT field view. Therefore, this new method was applied to improve the accuracy of the image fusion and treatment.

\section{Treatment interruption}

Each TMI treatment takes nearly $2.5 \mathrm{~h}$, which is a huge challenge for patients. During the course of treatment, if any urgent symptoms occur, such as vomiting, fever, convulsions, treatment must be stopped immediately. Because of large dose fraction (4-5 Gy/fraction) for the total body irradiation in our study, nearly half of patients develop acute radioactive reaction, especially in the second fraction. With these reasons, the risk team gave the interruption failure an $\mathrm{O}$ score of 8.5 and RPN value of 204.8. After group discussion, we recommended that sedative, tropisetron and antipyretic should be used more often to reduce adverse symptoms, before first
FMEA, sedative was only used for little child, who couldn't stay still during treatment.

\section{Second FMEA results}

Second FMEA was performed for the high-risk FMs based on the same risk analysis team in 1 year later. Thirty-two patients were treated with HT-TMI in this year. The second RPN value of the top 5 high-risk FMs were all decreased (details shown in Table 3). For the treatment couch movement failure, we tested the accuracy of the treatment couch before every treatment and found four times has exceeded the tolerance, treatment started until the treatment couch was repaired by the engineer. D score of the FM has dropped to 3.2 and RPN of 102.8. In the section plan dose junction error, an additional MVCT scan covering the dose junction region was performed to complement the the setup error in SI direction from Plan-upper and Plan-lower and found three times the shifts in SI direction has exceeded 10 $\mathrm{mm}$ in this year, if without complement, the dose of the junction region could be seriously underdose or overdose. So the risk analysis team gave the FM with an D score of 3.1 and RPN of 110.4. For the setup check by MVCT failure, standard three short scans rules was applied and six patients have been re-setup again, both $\mathrm{O}$, $\mathrm{D}$ and RPN score have dropped to 3.3, 3.5 and 94.6. For the patient immobilization error, after the new method of immobilizing the shoulders and arms was used, all the patients' PTVs in shoulders and arms could be seen in the MVCT images. So the O score was assigned with 3.2 and the final RPN was 90.2. The probability of treatment interruption was also decreased after sedative, tropisetron and antipyretic was used more often with an $\mathrm{O}$ score of 5.5 and RPN of 134.2. The top 5 high-risk FMs found in the first FMEA were all decreased and no longer high-risk FMs in the second FMEA after adaption of the QM program. A total of 20 new high-risk FMs were found and appropriate QM program were implemented based on the fault tree analysis.

\section{Discussion}

This paper used FMEA approach two times separated by 1 year to systematically uncover and analyze major FMs in a high risk clinical procedure - total marrow irradiation delivered with HT and propose feasible mitigations for the highest risk in the process.

In our study, the multidisciplinary team has a total of 20 members and not only consisted of a manager of the Medical Quality Control Committee of our department, a chief physicist and a chief therapist but also the regular physicians, physicists and therapists who participated in the daily clinical treatment process with varies number of years of working experience, which could make the 
FMs more comprehensive and close to the clinical procedure [24].

HT-TMI is a time consuming procedure with high precision, which puts high pressure on the machine and patient $[4,5]$. The treatment couch movement failure was assigned with the highest RPN, although quality assurance methods of the couch have been supported by some literature [23], the distance of couch movement test was only $20 \mathrm{~cm}$, which can not meet the demand of HT-TMI. For patient, the long treatment time was also a huge challenge, the treatment interruption failure was the fifth top FM, although Taiki Magome et al. [25] noted the fast megavoltage computed tomography approach can be used to shorten the scanning and image registration time, the treatment time can no longer be compressed due to the delivery mode of tomotherapy.

TMI is typically treated in 2 parts: an upper (body) and lower (legs) section, dose homogeneity at the junction region should be considered from the overlap of the two plans. Timothy E. Schultheiss et al. [4] has reported the dose junction method between tomotherapy and conventional linac, but the dose of the overlap region was $50 \%$ isodoses from the 2 treatment modalities. Mancosu et al. [26] has demonstrated the approach to create field junction from two volumetric modulated arc therapy plans with different patient orientation and obtained optimal target coverage of the treatment of TMI-TMLI. In our study, to complement the dose distributions of junction region from Plan-upper, in the Plan-lower delivery stage, the shifts in SI direction were set to match the setup error in SI direction from Plan-upper, which could be more accurate, but demand more steps and care. In terms of an FMEA approach, the method proposed by Mancosu et al. [26] may have the lower $\mathrm{O}$ and $\mathrm{S}$ scores of the dose junction error, because differences $<1 \%$ were found for mean doses to PTV and surrounding healthy tissue in the three directions regarding the 5 $\mathrm{mm}$ shifts. In our study, if the three consecutive slices from the Plan-upper and Plan-lower mismatched for 5 $\mathrm{mm}$, which could generate $110 \%$ hot-spots near the junction region.

Many centers use different immobilization and pretreatment position verification techniques. Two methods of pretreatment imaging were used: whole body MVCT imaging and partial body MVCT imaging [27]. But in fact, if the patient was not setup perfectly, often one whole body MVCT imaging or three scans can not reduce the setup errors well. In our study, three short scans were used to assess the whole body alignment in the early setup stage, if necessary, the patient should be re-setup. The new and feasible method of immobilizing the shoulders and arms was applied to improve the accuracy of the image fusion and treatment, which could effectively minimize the shoulder misalignment reported by Zuro et al. [27] .
In our study, we creatively performed FMEA two times separated by one year to verify the effectiveness of the QC program and found that the RPN value of the high-risk FM were all dropped. The QC program introduced by the first time FMEA did help us solve some of the high-risk FMs and the second FMEA has found some new high-risk FMs. Two or more times FMEA can help us to build the PDCA cycle (plan do check act cycle) and improve the QM program continuously.

HT-TMI is an uncommon technique for many radiotherapy centers, but the complexity and potential errors of it should not be ignored. After performing the FMEA, we have uncovered several high-risk steps in our process and redesigned the QC method, although the results may not apply to other centers who have conducted TMI in other way or even in the same way, the findings of the section plan dose junction technique and immobilization methods of shoulders and arms could be useful for centers treated TMI with HT.

Our study had several limitations. One was that the study was a single group analyzing an uncommon procedure. The results might not apply to others. To minimize this shortcoming, we enlarged the group numbers to a total of 20 people and cooperated with other centers that have the similar HT-TMI process. Second was that some solutions to particular failure modes added additional tasks to the process. For example, additional MVCT scan could lead new errors. Therefore, to properly use the results of FMEA, we should combine with the fault tree analysis to make the errors more visible to implement efficient QC method and try to not modify the process too much.

\section{Conclusion}

It can be concluded that QC interventions were implemented based on the FMEA results. The HT-TMI specific treatment couch tests; arms immobilization methods and strategy of section plan dose junction could be used to improve the safety.

\section{Abbreviations}

AP: anterior and posterior; C1: first cervical vertebra; CT: computed tomography; D: detectability; FFS: foot first superior; FM: failure mode; FMEA: failure mode and effects analysis; HT-MVCT: megavoltage computed tomography of helical tomotherapy; HT-TMI: total marrow irradiation treated with helical tomotherapy; L5: the fifth lumbar vertebra; LR: left and right; MVCT: megavoltage computed tomography; O: occurrence; PDCA: plan do check act; QC: quality control; QM: quality management; RPN: risk priority number; S: severity of impact; SI: superior and inferior; T10: the tenth thoracic vertebra; T7: the seventh thoracic vertebra; TMI: total marrow irradiation

\section{Acknowledgements}

The authors are grateful to all the colleagues in the Department of Radiation and Medical Oncology, Zhongnan Hospital of Wuhan University, Wuhan, China, for their assistance with the project. The authors thank An Liu for his assistance in editing this article. 


\section{Authors' contributions}

JS and XW conceived of this study and wrote the article. DD, JG, KT, HZ, ZB and JX participated in data collection. CX and HL supported the research and provided ideas. $Y Z$ and $\mathrm{AL}$ guided the paper revision. All authors read and approved the final manuscript.

\section{Funding}

No.

\section{Availability of data and materials}

Please contact author for data requests.

\section{Ethics approval and consent to participate}

This study was approved by the Regional Ethics Committee of Zhongnan Hospital of Wuhan University and all patients signed informed consents before treatment.

\section{Consent for publication}

Not applicable.

\section{Competing interests}

The authors declare that they have no competing interest.

\section{Author details}

'Department of Radiation and Medical Oncology, Zhongnan Hospital of Wuhan University, 169 Donghu Road, Wuhan, Hubei 430070, People's Republic of China. ${ }^{2}$ Hubei Radiotherapy Quality Control Center, Wuhan University, Wuhan, Hubei, China. ${ }^{3}$ Divisions of Radiation Oncology, City of Hope National Medical Center, Duarte, CA, USA.

Received: 23 September 2019 Accepted: 29 November 2019 Published online: 27 December 2019

\section{References}

1. Blaise D, Maraninchi D, Michallet. Long-term follow-up of a randomized trial comparing the combination of cyclophosphamide with total body irradiation or busulfan as conditioning regimen for patients receiving HLA identical marrow grafts for acute myeloblastic leukemia in first complete remission. Blood. 2001;97:3669-71.

2. Bunin N, Aplenc R, Kamani N. Randomized trial of busulfan vs total body irradiation containing conditioning regimens for children with acute lymphoblastic leukemia: a pediatric blood and marrow transplant consortium study. Bone Marrow Transplant. 2003;32:543-8.

3. Rosenthal J, Wong J, Stein A. Phase 1/2 trial of total marrow and lymph node irradiation to augment reduced-intensity transplantation for advanced hematologic malignancies. Blood. 2011:117:309-15.

4. Schultheiss TE, Wong J, Liu A. Image-guided total marrow and total lymphatic irradiation using helical tomotherapy. Int J Radiat Oncol Biol Phys. 2007;67:1259-67

5. Gruen A, Ebell W, Wlodarczyk W. Total body irradiation (TBI) using helical Tomotherapy in children and young adults undergoing stem cell transplantation. Radiat Oncol. 2013;8:92.

6. Wong JYC, Rosenthal J, Liu A. Total-marrow irradiation using helical Tomotherapy in patients with multiple myeloma and acute leukemia undergoing hematopoietic cell transplantation. Int J Radiat Oncol Biol Phys. 2009;73:273-9

7. Zeverino M, Agostinelli S, Taccini G. Advances in the implementation of helical tomotherapy-based total marrow irradiation with a novel field junction technique. Med Dosim. 2012;37:314-20

8. Penagaricano JA, Chao M, Van Rhee F. Clinical feasibility of TBI with helical tomotherapy. Bone Marrow Transplant. 2011;46:929-35.

9. Bissonnette JP, Medlam G. Trend analysis of radiation therapy incidents over seven years[J]. Radiother Oncol. 2010;96(1):139-44.

10. Cunningham J, Coffey M, Knoos T. Radiation oncology safety information system (ROSIS) - profiles of participants and the first 1074 incident reports. Radiother Oncol. 2010;97(3):601-7.

11. Williamson JF, Dunscombe PB, Sharpe MB. Quality assurance needs for modern image-based radiotherapy: recommendations from 2007 interorganizational symposium on quality assurance of radiation therapy: challenges of advanced technology. Int J Radiat Oncol Biol Phys. 2007:71: S2-S12. https://doi.org/10.1016/j.jirobp.2007.08.080.
12. Scorsetti M, Signori C, Lattuada $P$, et al. Applying failure mode effects and criticality analysis in radiotherapy: lessons learned and perspectives of enhancement. Radiother Oncol. 2010;94(3):367-74. https://doi.org/10.1016/j. radonc.2009.12.040.

13. Ford EC, Smith K, Terezakis S, et al. A streamlined failure mode and effects analysis. Med Phys. 2014;41:617-96. https://doi.org/10.1118/1.4875687.

14. Broggi S, Cantone MC, Chiara A. Application of failure mode and effects analysis (FMEA) to pretreatment phases in tomotherapy. J Appl Clin Med Phys. 2013;14(5):265-77. https://doi.org/10.1120/jacmp.v14i5.4329.

15. Jones RT, Handsfield L, Read PW. Safety and feasibility of STAT RAD: improvement of a novel rapid tomotherapy-based radiation therapy workflow by failure mode and effects analysis. Pract Radiat Oncol. 2015;5(2): 106-12. https://doi.org/10.1016/j.prro.2014.03.016.

16. Veronese I, De Martin E, Martinotti AS. Multi-institutional application of failure mode and effects analysis (FMEA) to cyberknife stereotactic body radiation therapy (SBRT). Radiat Oncol. 2015;10:132. https://doi.org/10.1186/ s13014-015-0438-0.

17. Manger RP, Paxton AB, Pawlickiand T, Kim G-Y. Failure mode and effects analysis and fault tree analysis of surface image guided cranial radiosurgery. Med Phys. 2015;42(5):2449-61. https://doi.org/10.1118/1.4918319.

18. Zheng $Y$, Johnson $R$, Larson G. Minimizing treatment planning errors in proton therapy using failure mode and effects analysis. Med Phys. 2016; 43(6):2904. https://doi.org/10.1118/1.4948686.

19. Cantone MC, Ciocca M, Dionisi F. Application of failure mode and effects analysis to treatment planning in scanned proton beam radiotherapy. Radiat Oncol. 2013:8:127. https://doi.org/10.1186/1748-717X-8-127.

20. Sayler L, Eldredge-Hindy H, Dinome J. Clinical implementation and failure mode and effects analysis of HDR skin brachytherapy using Valencia and Leipzig surface applicators. Brachytherapy. 2015;14(2):293-9. https://doi.org/ 10.1016/j.brachy.2014.11.007.

21. Lopez-Tarjuelo J, Bouche-Babiloni A, Santos-Serra A. Failure mode and effect analysis oriented to risk-reduction interventions in intraoperative electron radiation therapy: the specific impact of patient transportation, automation, and treatment planning availability. Radiother Oncol. 2014;113(2):283-9. https://doi.org/10.1016/j.radonc.2014.11.012

22. Bao Z, Zhao H, Wang D. Feasibility of a novel dose fractionation strategy in TMI/ TMLI. Radiat Oncol. 2018;13(1):248. https://doi.org/10.1186/s13014-018-1201-0.

23. Langen KM, Papanikolaou N, Balog J. QA for helical tomotherapy: report of the AAPM task group 148. Med Phys. 2010;37(9):4817-53. https://doi.org/10. 1118/1.3462971.

24. Saiful Huq M, Fraass BA, Dunscombe. The report of task group 100 of the AAPM: application of risk analysis methods to radiation therapy quality management. Med Phys. 2016:43:4209-62. https://doi.org/10.1118/1.4947547.

25. Magome T, Haga A, Takahashi Y. Fast megavoltage computed tomography: a rapid imaging method for Total body or marrow irradiation in helical Tomotherapy. Int J Radiat Oncol Biol Phys. 2016;96(3):688-95. https://doi. org/10.1016/j.jijrobp.2016.06.2458.

26. Mancosu P, Navarria P, Castagna L. Plan robustness in field junction region from arcs with different patient orientation in total marrow irradiation with VMAT. Phys Med. 2015;31(7):677-82. https://doi.org/10.1016/j.ejmp.2015.05.012.

27. Zuro D, Vagge S, Broggi S. Multi-institutional evaluation of MVCT guided patient registration and dosimetric precision in total marrow irradiation: a global health initiative by the international consortium of total marrow irradiation. Radiother Oncol. 2019. https://doi.org/10.1016/..radonc.2019.07.010.

\section{Publisher's Note}

Springer Nature remains neutral with regard to jurisdictional claims in published maps and institutional affiliations. 